EPJ Web of Conferences 97,00030 (2015)

DOI: $10.1051 /$ epjconf/ 20159700030

(C) Owned by the authors, published by EDP Sciences, 2015

\title{
Latest results from EPOS3 on the production of stable and un- stable hadrons
}

\author{
K. Werner ${ }^{1}$, B. Guiot ${ }^{1}$, Iu. Karpenko ${ }^{2,3}$, A.G. Knospe ${ }^{4}$, C. Markert ${ }^{4}$, T. Pierog ${ }^{5}$, and J. Steinheimer ${ }^{3}$ \\ ${ }^{1}$ SUBATECH, University of Nantes - IN2P3/CNRS- EMN, Nantes, France \\ ${ }^{2}$ Bogolyubov Institute for Theoretical Physics, Kiev 143, 03680, Ukraine \\ ${ }^{3}$ FIAS, Johann Wolfgang Goethe Universitaet, Frankfurt am Main, Germany \\ ${ }^{4}$ The University of Texas at Austin, Physics Department, Austin, TX, USA \\ ${ }^{5}$ Karlsruhe Inst. of Technology, KIT, Campus North, Inst. f. Kernphysik, Germany
}

\begin{abstract}
Evidence for hydrodynamical flow in AA or in pA collisons is to a large extent obtained from the observation of identified hadrons, such as pions, kaons, and protons. But much more information in particular about the late stage can be obtained by also considering unstable particles, which decay during the lifetime of the expanding hadronic matter. We therefore started to use EPOS3, a unified approach for pp, pA, and AA scattering, to investigate the production of stable and unstable particles.
\end{abstract}

\section{The unified approach}

Collective hydrodynamic flow seems to be well established in heavy ion (HI) collisions at energies between 200 and $2760 \mathrm{AGeV}$, whereas p-p and p-A collisions are often considered to be simple reference systems, showing "normal" behavior, such that deviations of HI results with respect to p-p or p-A reveal "new physics". Surprisingly, the first results from $\mathrm{p}-\mathrm{Pb}$ at $5 \mathrm{TeV}$ on the transverse momentum dependence of azimuthal anisotropies and particle yields are very similar to the observations in HI scattering $[1,2]$. We take these experimental observations as an excellent motivation and justification for a unified description of the dynamics of ALL reactions, from p-p to AA [3]. In this picture, the same procedure applies, referred to as EPOS3 [4], based on several stages:

Initial conditions. A Gribov-Regge multiple scattering approach is employed [5], where the elementary object (by definition called Pomeron) is a DGLAP parton ladder, using in addition a CGC motivated saturation scale [6] for each Pomeron, of the form $Q_{s} \propto N_{\text {part }} \hat{s}^{\lambda}$, where $N_{\text {part }}$ is the number of nucleons connected the Pomeron in question, and $\hat{s}$ its energy. The parton ladders are treated as classical relativistic (kinky) strings.

Core-corona approach. At some early proper time $\tau_{0}$, one separates fluid (core) and escaping hadrons, including jet hadrons (corona), based on the momenta and the density of string segments $[4,7]$. The corresponding energy-momentum tensor of the core part is transformed into an equilibrium one, needed to start the hydrodynamical evolution. This is based on the hypothesis that equilibration happens rapidly and affects essentially the space components of the energy-momentum tensor.

This is an Open Access article distributed under the terms of the Creative Commons Attribution License 4.0, which permits unrestricted use, distribution, and reproduction in any medium, provided the original work is properly cited. 


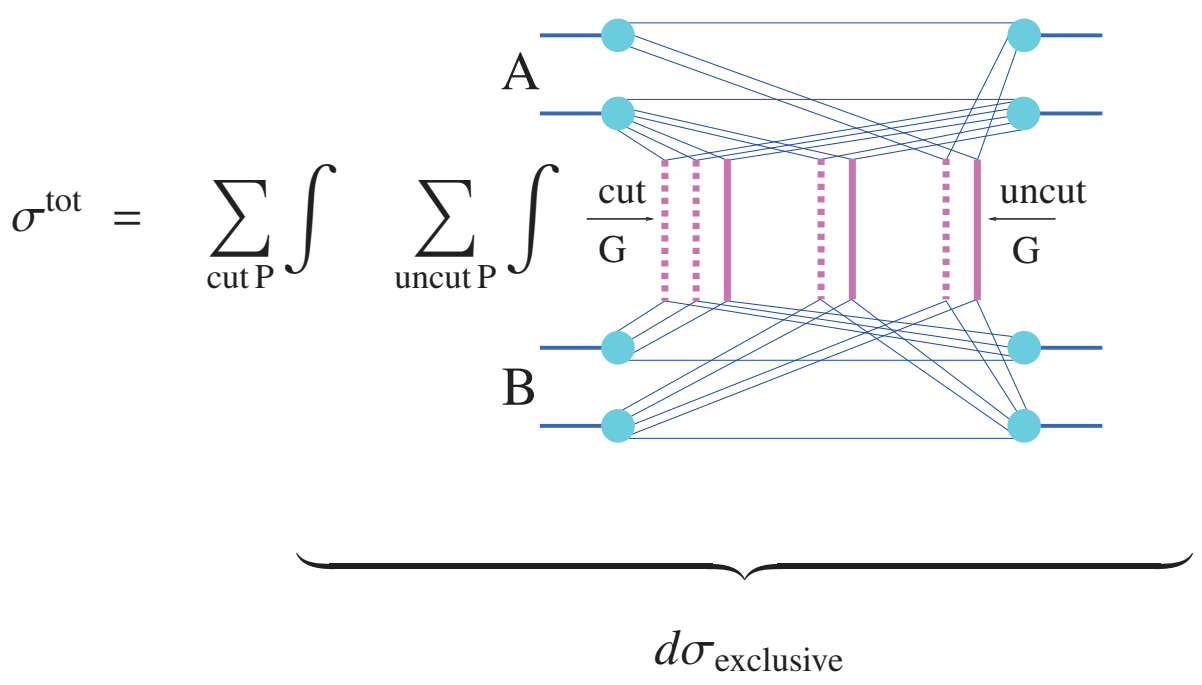

Figure 1. (Color online) PBGRT formalism: The total cross section expressed in terms of cut (dashed lines) and uncut (solid lines) Pomerons, for nucleus-nucleus, proton-nucleus, and proton-proton collisions. Partial summations allow to obtain exclusive cross sections, the mathematical formulas can be found in [5], or in a somewhat simplified form in [4].

Viscous hydrodynamic expansion. Starting from the initial proper time $\tau_{0}$, the core part of the system evolves according to the equations of relativistic viscous hydrodynamics $[4,8]$, where we use presently $\eta / s=0.08$. A cross-over equation-of-state is used, compatible with lattice QCD [9, 10].

Statistical hadronization The "core-matter" hadronizes on some hypersurface defined by a constant temperature $T_{H}$, where a so-called Cooper-Frye procedure is employed, using equilibrium hadron distributions, see [10].

Final state hadronic cascade After hadronization, the hadron density is still big enough to allow hadron-hadron rescatterings. For this purpose, we use the UrQMD model [11].

The above procedure is employed for each event (event-by-event procedure).

Whereas our approach is described in detail in [4], referring to older works [5, 7, 10], we confine ourselves here to a couple of remarks, to selected items. The initial conditions are generated in the Gribov-Regge multiple scattering framework. Our formalism is referred to as "Parton-Based GribovRegge Theory" (PBGRT) and described in very detail in [5], see also [4] for all the details of the present (EPOS3) implementation. The fundamental assumption of the approach is the hypothesis that the S-matrix is given as a product of elementary objects, referred to as Pomerons. Once the Pomeron is specified (taken as a DGLAP parton ladder, including a saturation scale), everything is completely determined. Employing cutting rule techniques, one may express the total cross section in terms of cut and uncut Pomerons, as sketched in fig. 1. The great advantage of this approach: doing partial summations, one obtains expressions for partial cross sections $d \sigma_{\text {exclusive }}$, for particular multiple scattering configurations, based on which the Monte Carlo generation of configurations can 

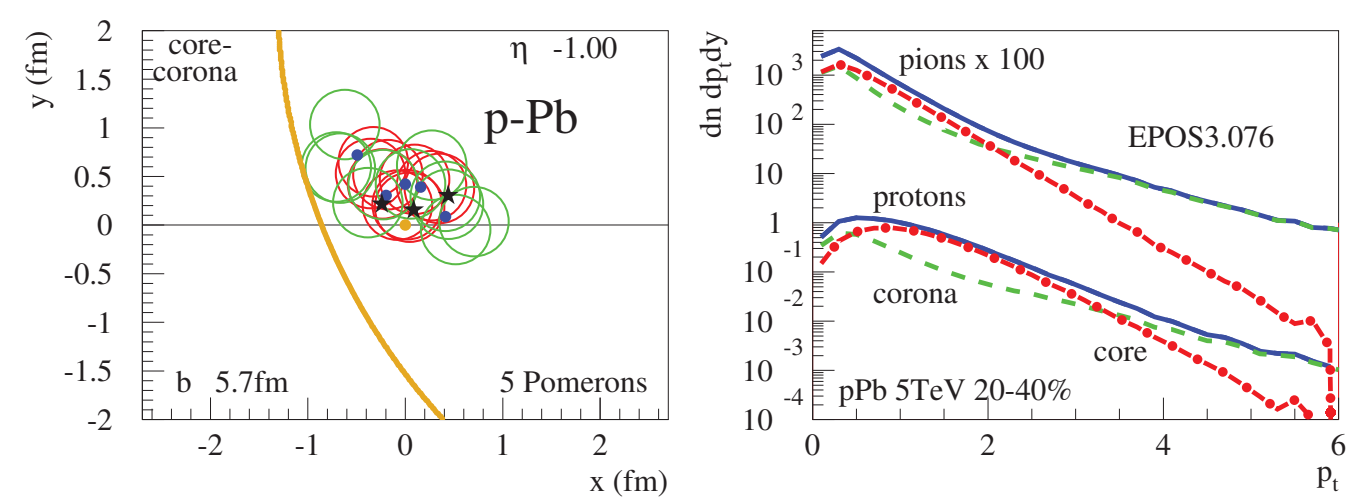

Figure 2. (Color online) Left: Core-corona separation in a semi-peripheral p-Pb collision. Right: the corresponding core and corona contributions to the $p_{t}$ spectra of pions and protons.

be done. No additional approximations are needed. The above multiple scattering picture is used for p-p, p-A, and A-A.

Based on the PBGRT approach, we obtain in A-A collisions a very large number of strings, but the randomness of their transverse positions leads to "bumpy" energy density distributions in the transverse plane at $\tau_{0}$, as published for the first time in the year 2000, see fig. 21 of [12]. This randomness of the initial distributions (in p-p, p-A, and A-A) in the transverse plane is the origin of azimuthal asymmetries in the distributions of the final particles, which are conveniently summarized in terms of flow harmonics $v_{n}$.

Our multiple scattering approach leads in a natural way to very simple features when it comes to relating soft and hard particle production. Be $N_{\text {hard }}$ the multiplicity of some "hard" particle production (like the $D$ meson multiplicity) and $N_{\text {ch }}$ the usual charged particle multiplicity in some phase space interval. We expect to first approximation a linear relation, $N_{\text {hard }} \propto N_{\mathrm{ch}}$, since both are proportional to the number $N_{\text {Pom }}$ of Pomerons. We obtain indeed such a linear behavior, as shown in [3], in perfect agreement with experimental data [13].

To understand the results later in this paper, we will discuss an example of core-corona separation in a semi-peripheral p-Pb collision, as shown in fig. 2. Shown (in the left figure) are string segments in the transverse plane, red (core) and green (corona) ones. There are sufficient overlapping core string segments to provide a core of plasma matter, showing a (short) hydrodynamic expansion, quickly building up flow. In the right figure, we plot the contribution from core and corona to the $p_{t}$ spectra of pions and protons. In particular for protons, the core dominates at intermediate $p_{t}$ (mass effect).

\section{Reaction scenarios and analysis methods}

In order to better understand particle production in EPOS, we will compare different scenarios, as there are: 
(A) No_cascade, no_decays refers to "basic EPOS" (including hydro evolution and hadronization), without decays and without hadronic cascade.

(B) No cascade, with decays refers to the previous case, but here resonance decays are done.

(C) Full (cascade \& decays) is the"full" scenario, namely "basic EPOS" (including hydro evolution and hadronization) plus the subsequent UrQMD hadronic cascade, where hadronic interactions and decays are realized.

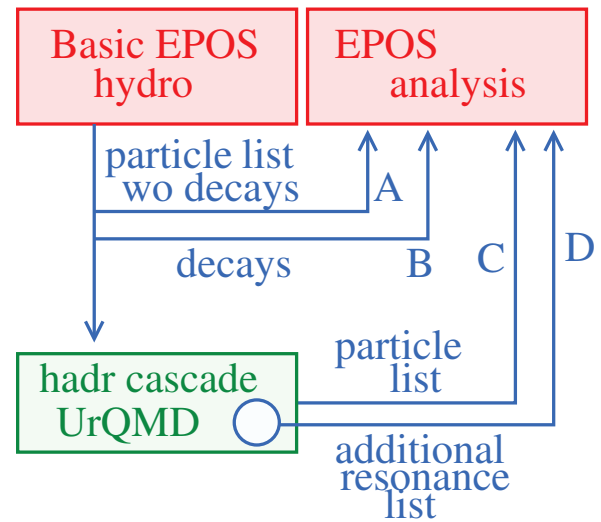

All these three scenarios can be realized in parallel in a single simulation run, the corresponding particle lists being stored into ROOT tree structures, which maybe analyzed online (during the simulation runs) or offline (reading in the previously produced ROOT files, where the user may use his own analysis tools).

In addition to these particle lists, thanks to a new program unit, we track all resonance decays inside UrQMD, and create an additional list of particles (resonances), including information about the decay modes the fate of the decay daughters. We distinguish between resonances where the daughters

Table 1. Meson resonances and decay modes, being monitored during the rescattering process.

\begin{tabular}{|c|c|c|}
\hline Code & Resonance & Daughters \\
\hline \hline 11100 & rho0 & pi- $^{-}$pi+ \\
\hline-12100 & rho- & pi-, pi0 \\
\hline 12100 & rho+ & pi+, pi0 \\
\hline 23100 & $\mathrm{~K}^{*} 0$ & pi-, K+ \\
\hline 23101 & $\mathrm{~K}^{*} 0$ & pi0, $\mathrm{K} 0$ \\
\hline
\end{tabular}

\begin{tabular}{|c|c|c|}
\hline Code & Resonance & Daughters \\
\hline \hline 13100 & $\mathrm{~K}^{*}+$ & pi+, K0 \\
\hline 13101 & $\mathrm{~K}^{*}+$ & pi0, $\mathrm{K}+$ \\
\hline 33100 & phi & $\mathrm{K}+, \mathrm{K}-$ \\
\hline 33101 & phi & $\mathrm{K} 0, \mathrm{~K} 0 \mathrm{bar}$ \\
\hline
\end{tabular}

Table 2. Baryon resonances and decay modes, being monitored during the rescattering process.

\begin{tabular}{|c|c|c|}
\hline Code & Resonance & Daughters \\
\hline \hline 123000 & Sigma0 & photon , Lambda \\
\hline 123400 & Lambda(1520) & K-, proton \\
\hline 123401 & Lambda(1520) & anti-K0, $\mathrm{n}$ \\
\hline 123401 & Lambda(1520) & $\mathrm{n}$, anti-K0 \\
\hline 123402 & Lambda(1520) & pi- , Sigma+ \\
\hline 123403 & Lambda(1520) & pi,+ Sigma- \\
\hline 222100 & Delta(1232)- & pi- , neutron \\
\hline 122100 & Delta(1232)0 & pi-, proton \\
\hline 122101 & Delta(1232)0 & pi0, neutron \\
\hline
\end{tabular}

\begin{tabular}{|c|c|c|}
\hline Code & Resonance & Daughters \\
\hline \hline 112100 & Delta(1232)+ & pi+, neutron \\
\hline 112101 & Delta(1232)+ & pi0, proton \\
\hline 111100 & Delta(1232)++ & pi+, proton \\
\hline 113100 & Sigma(1385)+ & pi+, Lambda \\
\hline 223100 & Sigma(1385)- & pi- , Lambda \\
\hline 233100 & Xi(1530)- & pi-, Xi0 \\
\hline 233101 & Xi(1530)- & pi0 , Xi- \\
\hline 133100 & $\mathrm{Xi}(1530) 0$ & pi+, Xi- \\
\hline 13310 & $\mathrm{Xi}(1530) 0$ & pi0 Xi0 \\
\hline
\end{tabular}


interact further via hadronic rescatterings or not. The list of resonances and decay modes considered presently is given in tables 1 and 2 .

\section{Particle spectra in $\mathrm{PbPb}$ at $2.76 \mathrm{ATeV}$}

In fig. 3, we show the different contributions to the transverse momentum spectra of charged particles at central rapidity in $\mathrm{PbPb}$ collisions, for different centralities. In scenario A (no cascade, no decays), for central collisions, the core dominates up to $4.5 \mathrm{GeV} / \mathrm{c}$, before the (hard) corona takes over. Going towards more peripheral scatterings, the relative weight of the corona contribution increases. But even for the most peripheral collisions, there is a substantial core contributions. In our framework, the hydrodynamic core even contributes in pp scattering, and very substantially in pA scattering.

The scenario C (full simulation) shows very similar results, but the core contribution is somewhat bigger. It should be said that during the rescattering process, whenever a core particle scatters, the outgoing hadrons are all considered to be core. So obviously the core can only increase during the rescattering.

In fig. 4, we do the same analysis, but this time considering only baryons. We observe roughly the same features as discussed above for the charged particles: core dominance at low $p_{t}$, and the corona takes over at high $p_{t}$.

What is different compared to the charged particle results, is the shape of the core contribution, showing a distinct maximum around $1-2 \mathrm{GeV} / \mathrm{c}$. This is due to the fact that baryons are on the average
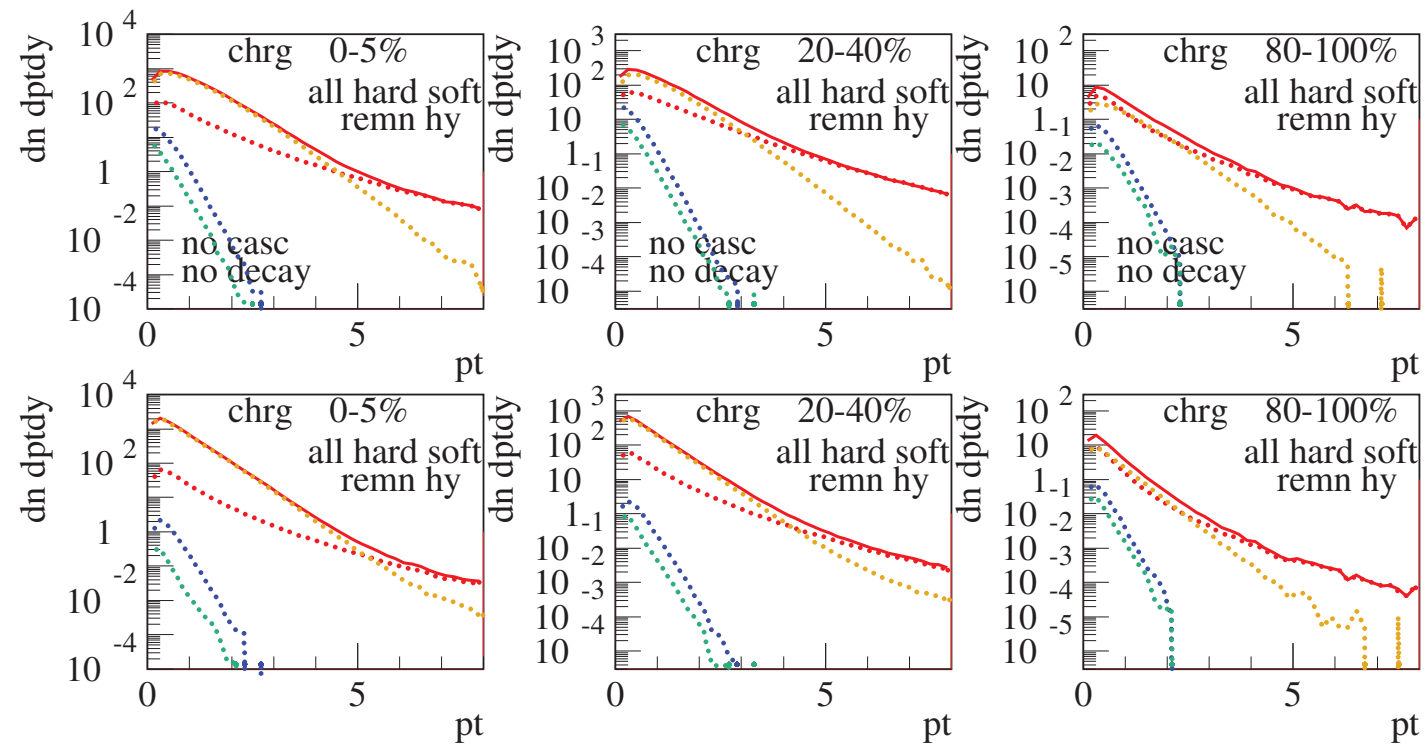

Figure 3. The different contributions to the transverse momentum spectra of charged particles at central rapidity in $\mathrm{PbPb}$ collisions, for different centralities. We consider the full scenario $(\mathrm{C})$ in the lower panel, and the scenario without cascade and without decays (A) in the upper panel. The most important contributions are the core (yellow dotted) and the (hard) corona (red dotted). Soft and remnant contributions are small. 

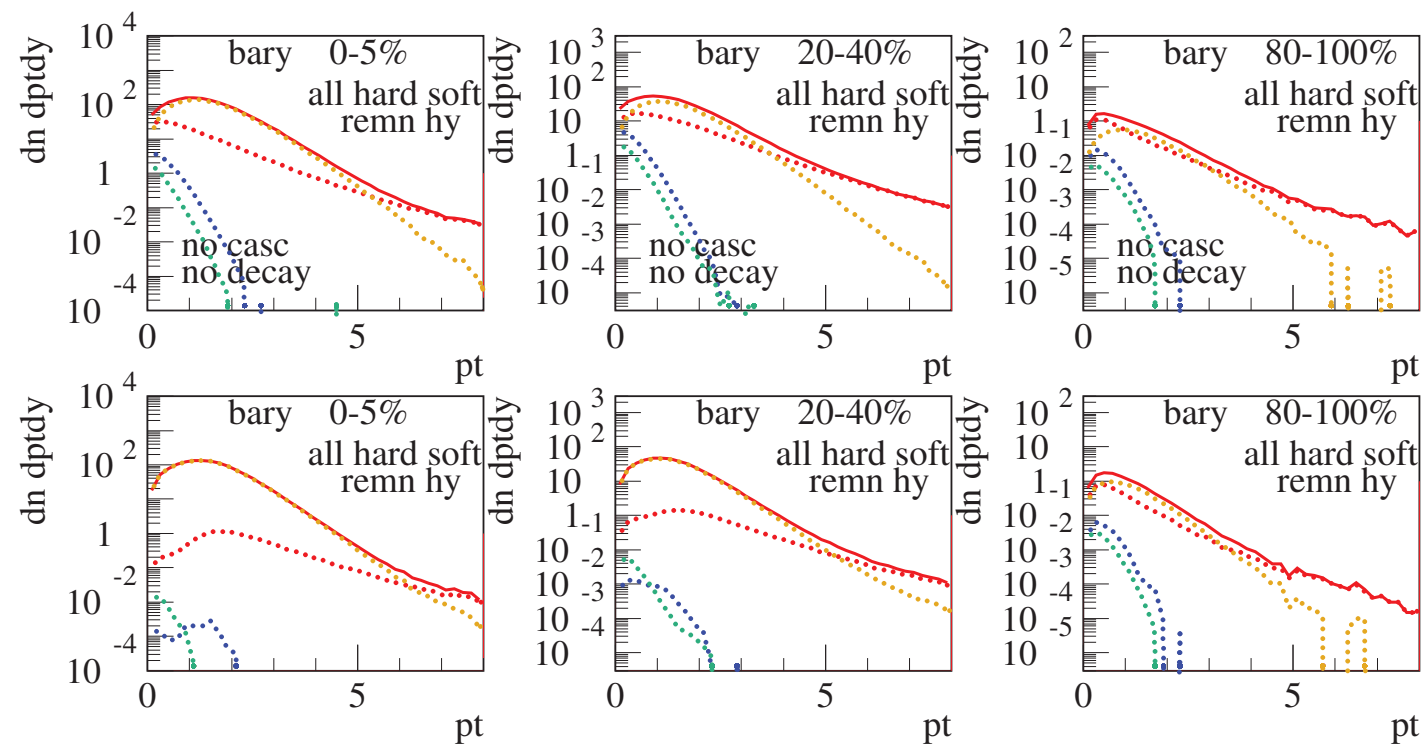

Figure 4. Same as fig. 3, but here we consider baryons.
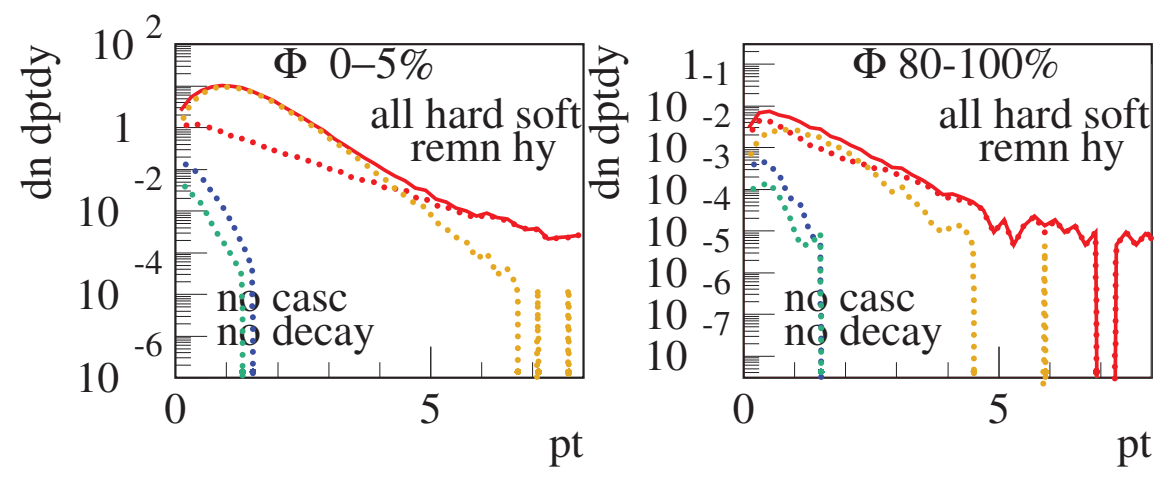

Figure 5. The different contributions to the transverse momentum spectra of $\phi$ mesons, for the scenario without cascade and without decays (A).

heavier, and they are therefore pushed to higher $p_{t}$ values, due to the hydrodynamical flow. Comparing the upper and the lower panel, for central collisions, we see a very strong hadronic rescattering activity, transforming almost all corona particles to core ones.

In fig. 5, we show the different contributions to the transverse momentum spectra of $\phi$ mesons, for the scenario without cascade and without decay (A). We see that the shapes are very similar compared 

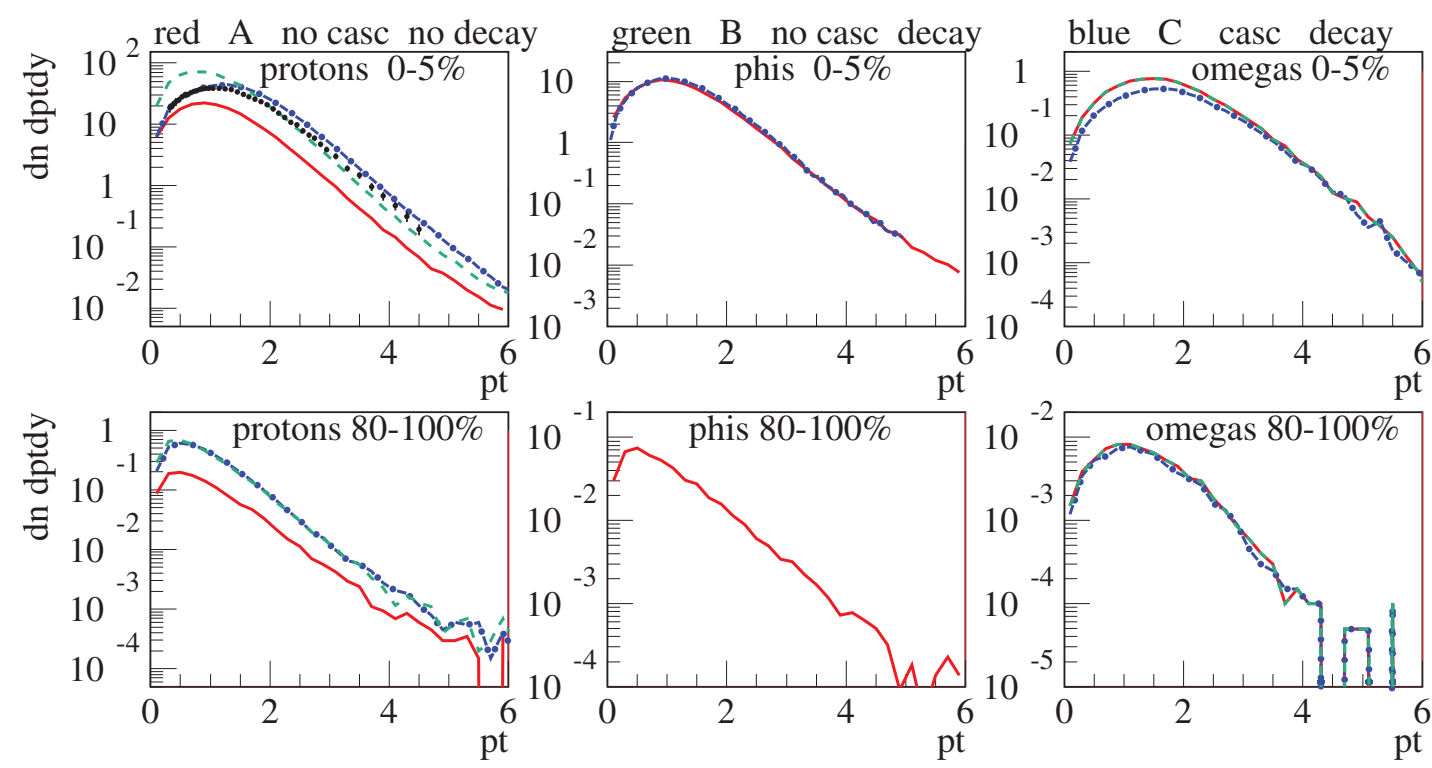

Figure 6. Transverse momentum spectra of protons (upper panel), $\phi$ mesons (middle panel), and $\Omega$ baryons (lower panel) at central rapidity in $\mathrm{PbPb}$ collisions, for different centralities. We consider the three scenarios $\mathrm{A}$ (no cascade no decay), B (no cascade decay), and C (full simulation,cascade decay), with solid red lines (A), dashed green lines (B), and dashed-dotted blue lines (C). The black dots are data from ALICE [14].

to the baryons (essentially protons), understandably due to the large mass of the $\phi$ meson, being close to the proton mass.

In fig. 6, we provide a more quantitative comparison of the different scenarios. Whereas from the previous plots it seems that $\phi$ mesons and baryons behave similarly, we see here that hadronic rescattering affects $\phi$ mesons and different baryons very differently. Since for the peripheral collisions the effect of rescattering is small, we discuss in the following central collisions. Comparing the blue curve with the green one for protons, we see a significant increase at high $p_{t}$, which means strong rescattering activity. The decrease at low $p_{t}$ is partly rescattering, partly annihilation. Very different situation for the $\phi$ mesons: There is only a slight reduction at low $p_{t}$ due to $\phi$ mesons which decay into daughters which then rescatter (we count here only $\phi$ mesons with surviving decay products). Concerning the $\Omega$ baryons, we see some annihilation affects at low $p_{t}$, whereas no change due to rescattering is visible at high $p_{t}$. In other words, the $\Omega$ baryons "freeze out" very early.

The latter observation will lead to some problem. Experimental data show a very pronounces mass splitting effect for $v_{2}$ as a function of $p_{t}$. To obtain such a mass splitting, one needs a long hadronic phase, an early freeze out will brake the mass ordering. See also the discussion in [16].

In fig. 7, we show $v_{2}$ as a function of $p_{t}$ for pions (red), protons (blue) and $\Omega$ baryons (green) for the $40-50 \%$ most central $\mathrm{PbPb}$ scatterings at $2.76 \mathrm{ATeV}$. The full lines are simulations, the dots data from ALICE [15]. Considering scenario B, without hadronic cascade (and therefore early freezeout), we observe some mass splitting, but not enough to explain the data. Including the hadronic cascade (right plot), pions and protons get sufficiently separated, but the omega curve does not follow the 

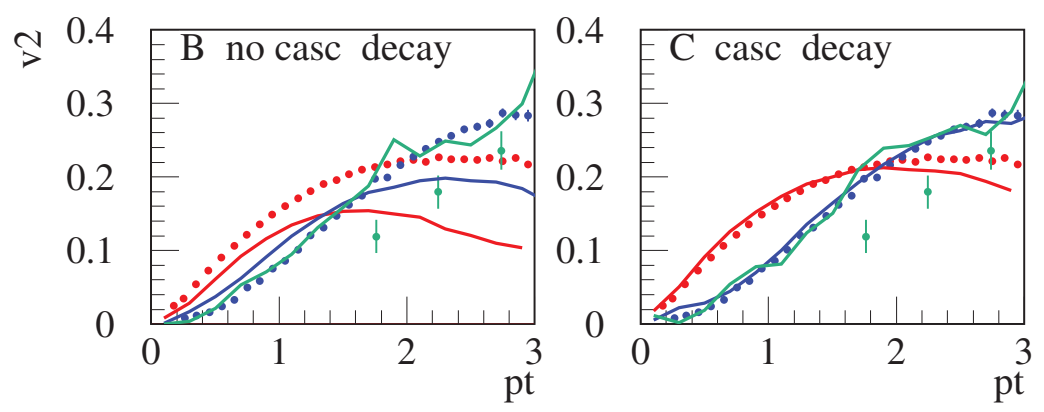

Figure 7. $v_{2}$ as a function of $p_{t}$ for pions (red), protons (blue) and $\Omega$ baryons (green) for the $40-50 \%$ most central $\mathrm{PbPb}$ scatterings at $2.76 \mathrm{ATeV}$. The full lines are simulations, the dots data from ALICE [15]. We show results for the scenario B (no cascade, including decays), and C (full simulation,including cascade and decays)

mass splitting trend seen in the data. Actually the omega curve is not much affected by rescattering, as expected from the earlier discussion.

To summarize: Recent developments allow us to make a detailed analysis of the production of stable and unstable hadrons within the EPOS3 approach. First studies show that already for such an elementary case as $\Omega$ production there seems to be a problem with the "standard model" of heavy ion physics (hydro + cascade), which is favored by other observations. It seems that an important piece is still missing.

Acknowledgements: This research was carried out within the scope of the GDRE (European Research Group) "Heavy ions at ultrarelativistic energies". Iu.K acknowledges support by the National Academy of Sciences of Ukraine (Agreement 2014) and by the State Fund for Fundamental Researches of Ukraine (Agreement 2014). Iu.K. acknowledges the financial support by the LOEWE initiative of the State of Hesse and Helmholtz International Center for FAIR. B.G. acknowledges the financial support by the TOGETHER project of the Region of "Pays de la Loire". The authors acknowledge the Texas Advanced Computing Center (TACC) at The University of Texas at Austin for providing HPC resources that have contributed to the research results reported within this paper. URL: http://www.tacc.utexas.edu.

\section{References}

[1] CMS collaboration, EPJC 74, 2847 (2014), arXiv:1307.3442

[2] ALICE collaboration, Phys. Lett. B 728, 25-38 (2014), arXiv:1307.6796

[3] K. Werner et al., proc of the XXIV International Conference on Ultrarelativistic NucleusNucleus Collisions (Quark Matter 2014), published in Nucl. Phys. A.

[4] K. Werner et al., Phys.Rev. C 89, 064903 (2014), arXiv:1312.1233

[5] H. J. Drescher, M. Hladik, S. Ostapchenko, T. Pierog and K. Werner, Phys. Rep. 350, 93 (2001)

[6] L. McLerran, R. Venugopalan, Phys. Rev. D 49, 2233 (1994); L. McLerran, R. Venugopalan, Phys. Rev. D 49, 3352 (1994); L. McLerran, R. Venugopalan, Phys. Rev. D 50, 2225 (1994)

[7] K. Werner, Phys. Rev. Lett. 98, 152301 (2007) 
[8] Iu. Karpenko, P. Huovinen, M. Bleicher, arXiv:1312.4160

[9] S. Borsanyi et al., JHEP 1011, 077 (2010), arXiv:1007.2580

[10] K. Werner, Iu. Karpenko, T. Pierog, M. Bleicher, K. Mikhailov, arXiv:1010.0400, Phys. Rev. C 83, 044915 (2011)

[11] M. Bleicher et al., J. Phys. G 25, 1859 (1999); H. Petersen, J. Steinheimer, G. Burau, M. Bleicher and H. Stocker, Phys. Rev. C 78, 044901 (2008)

[12] H.J. Drescher, S. Ostapchenko, T. Pierog, K. Werner, hep-ph/0011219, PhysRev C 65, 054902 (2002)

[13] Zaida Conesa Del Valle, ALICE, Fourth International Workshop on Multiple Partonic Interactions at the LHC, CERN, 2012

[14] ALICE collaboration, Phys. Rev. C 88, 044910 (2013), arXiv:1303.0737

[15] ALICE collaboration, arXiv: 1405.4632

[16] R. Snellings, this proceedings. 
